\title{
Recent Aspects of the Chondroprotective and
}

\section{Anti-Inflammatory Actions of Glucosamine, a Functional Food}

\author{
ISAO NAGAOKA* \\ *Department of Host Defense and Biochemical Research, Juntendo University, Graduate School of Medicine, Tokyo, Japan
}

\begin{abstract}
Glucosamine, a naturally occurring amino monosaccharide, is present in the connective and cartilage tissues as a component of glycosaminoglycans. Thus, glucosamine has been widely used to treat osteoarthritis, a joint disease characterized by cartilage degeneration, in humans. We previously revealed that glucosamine induces the production of hyaluronic acid by synovial cells and chondrocytes, and increases the expression of hyaluronic acid-synthesizing enzymes (HAS) in these cells, indicating that it exhibits chondroprotective action on osteoarthritis by modulating the expression of HAS and inducing the production of hyaluronic acid (a major component of glycosaminoglycans) by synovial cells and chondrocytes. Furthermore, we recently examined the expression of the sirtuin (SIRT) gene family in chondrocytes, and revealed that glucosamine significantly increased the mRNA and protein levels of SIRT1 (a putative gene involved in lifespan elongation) in chondrocytes, suggesting that glucosamine can upregulate the mRNA and protein levels of SIRT1 in chondrocytes, thereby possibly exhibiting protective action against osteoarthritis.

In addition, glucosamine is expected to exert anti-inflammatory action since it downregulates the expression of pro-inflammatory cytokines. We recently identified a transcription factor, Spl, as an $O$-linked-N-acetylglucosamine $(O-G l c N A c)$-modified protein. Namely, glucosamine enhanced the $O$-GlcNAc modification of Spl, and the effect was abolished by alloxan, an $O$-GlcNAc transferase inhibitor. Moreover, glucosamine downregulated the expression of $\mathrm{IL}-8$, and the effect was abrogated by alloxan. These observations apparently suggest that glucosamine modulates (suppresses) IL-8 expression via the $O$-GlcNAc modification of Sp1 in synovial cells.

Together, these observations indicate that glucosamine can be therapeutically utilized as a functional molecule with chondroprotective and anti-inflammatory actions in the body.
\end{abstract}

Key words: glucosamine, functional food, anti-inflammatory, chondroprotective, osteoarthritis

\section{What is glucosamine?}

Glucosamine is an amino monosaccharide with an amino group, and functioning as a component of glycosaminoglycans in our body ${ }^{1)}$ (Figure-1).

Glucosamine is mostly manufactured from chitin, a polymer of $\mathrm{N}$-acetyl-glucosamine present in the shells of shrimps and crab (Figure-2). Chitosan, a polymer of glucosamine is produced from the chitin by deacetylation under alkaline condition. In contrast, glucosamine, a chitosan monomer is produced as glucosamine hydrochloride by hydrolysis and deacetylation of chitin under acidic condi-

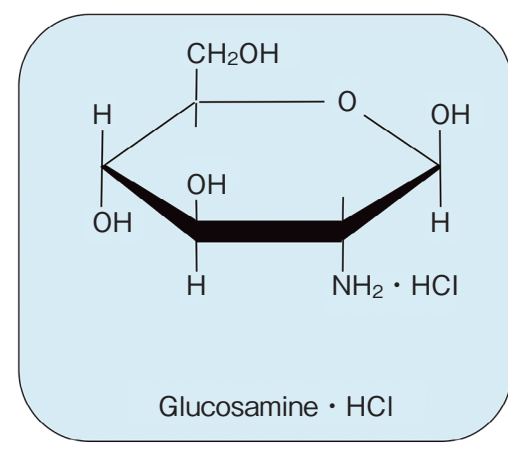

Amino monosachharide

A component of glycosaminoglycans

(e.g. hyaluronic acid) in our body

$\square$ A functional food with a chondroprotective action

Figure-1 Structure and functions of glucosamine

Isao Nagaoka

Department of Host Defense and Biochemical Research, Juntendo University, Graduate School of Medicine

2-1-1 Hongo, Bunkyo-ku, Tokyo 113-8421, Japan

TEL: +81-3-5802-1032 E-mail: nagaokai@juntendo.ac.jp

[Received Nov. 4, 2014] 


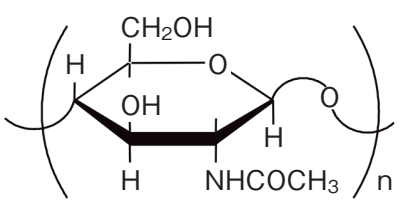

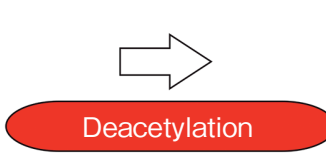

$\mathrm{NaOH}$

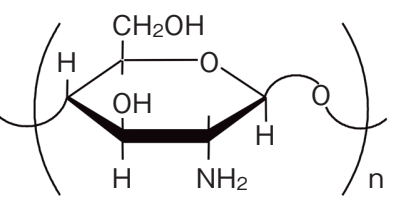

Chitosan

(glucosamine polymer)

Chitin

(N-acetyl-glucosamine polymer) in the shells of shrimps and crab
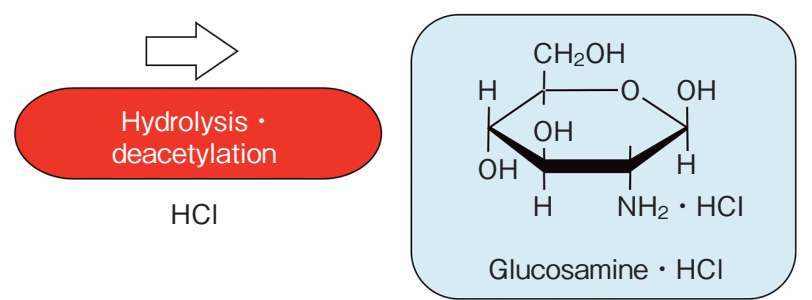

Figure-2 Manufacturing processes of chitosan (a glucosamine polymer) and glucosamine from chitin (an N-acetyl-glucosamine polymer)

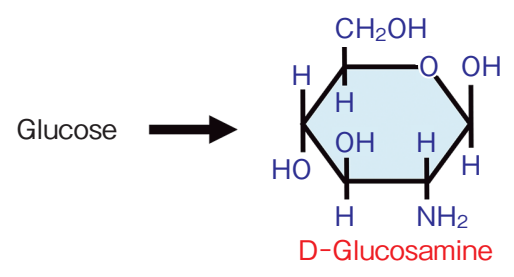

Hyaluronic acid

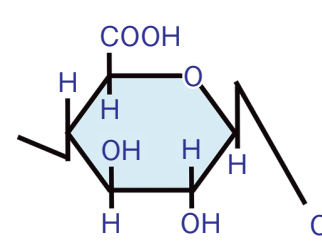

D-Glucuronic acid

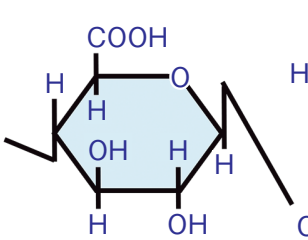

D-Glucuronic acid

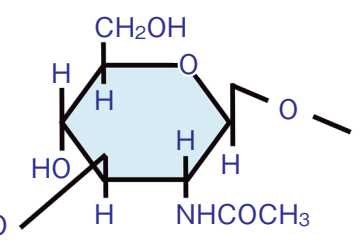

$\mathrm{N}$-Acetyl-D-glucosamine

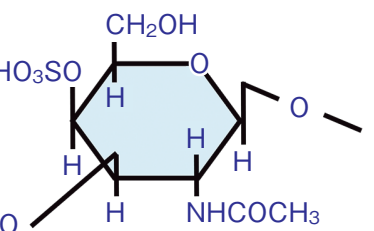

$\mathrm{N}$-Acetyl-D-

galactosamine-4-sulfate

Figure-3 Structures of glycosaminoglycans

tion. Figure- 3 shows the basic structures of glycosaminoglycans such as hyaluronic acid and chondroitin sulfate, which are composed of uronic acid (such as glucuronic acid) and glucosamine-derivatives (such as $\mathrm{N}$-acetyl-glucosamine and $\mathrm{N}$-acetyl-galactosamine).

Figure-4 shows the synthetic pathway of glycosaminoglycans ${ }^{2)}{ }^{3)}$. Glucose is converted into glucosamine with the addition of amino group from glutamine, and further converted into $\mathrm{N}$-acetyl-glu- cosamine and $\mathrm{N}$-acetyl-galactosamine. Then, these glucosamine-derivatives are coupled with uronic acid to form glycosaminoglycans present in the joint cartilage, skin and other tissues. Importantly, glucosamine supplement is efficiently absorbed from the intestine, delivered to several tissues and utilized as a component of glycosaminoglycans. Thus, glucosamine is widely utilized as a functional food with a chondroprotective action to treat human diseases such as osteoarthritis as a precur- 


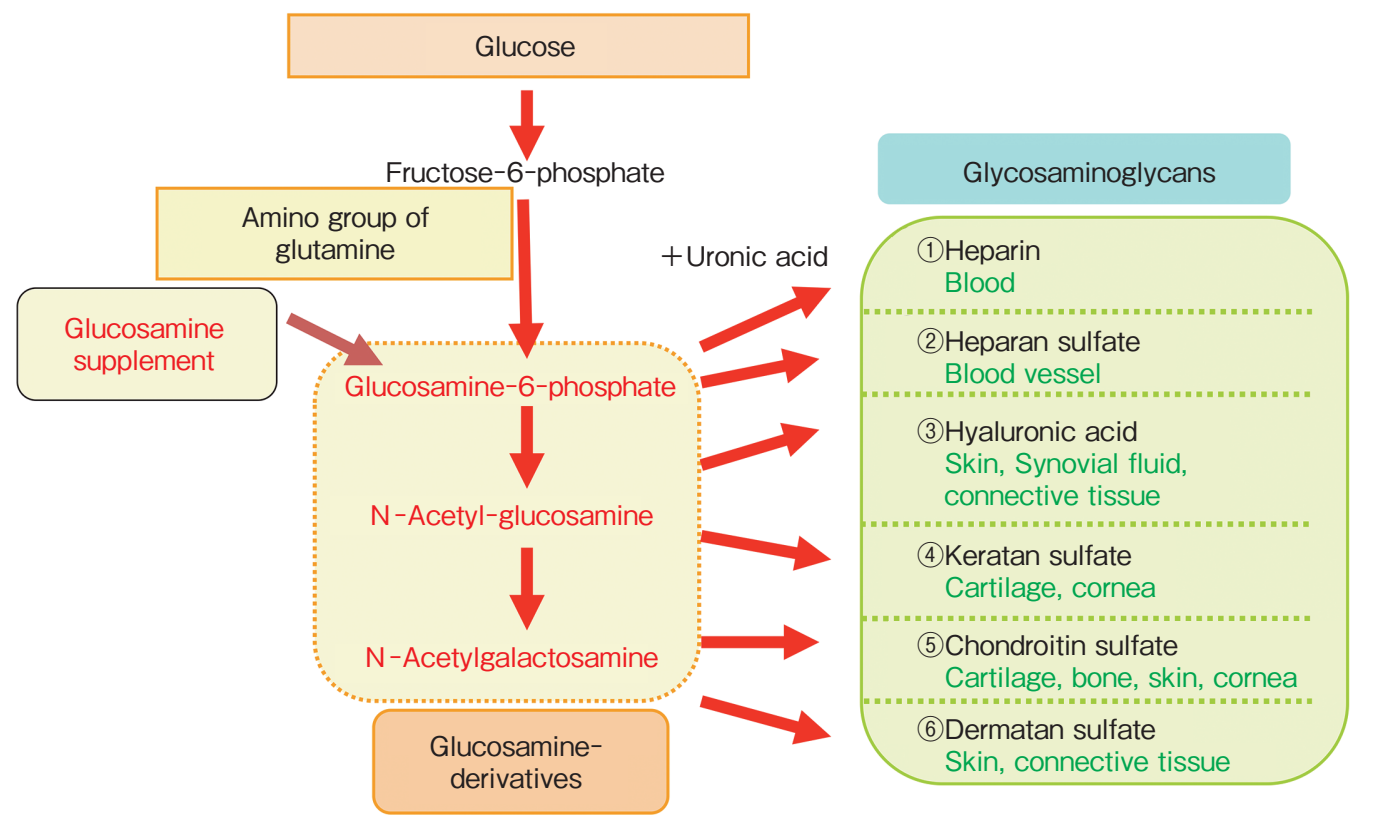

Figure-4 Synthesis of glycosaminoglycans from glucosamine-derives and uronic acid

sor of glycosaminoglycans ${ }^{4)-6}$.

In addition, we revealed that glucosamine suppresses the activation of neutrophils ${ }^{7)}$, synovial cells $^{8)}$ and intestinal epithelial cells ${ }^{9)}$, endothelial cells ${ }^{10)}$, and inhibits adjuvant arthritis ${ }^{11)}$, colitis ${ }^{12)}$ and atherosclerosis ${ }^{13)}$ in animal models. Thus, glucosamine is also expected to exert anti-inflammatory actions. Based on our recent findings, the chondroprotective and anti-inflammatory actions of glucosamine are discussed in this review.

\section{Chondroprotective actions of glucosamine}

Osteoarthritis is a joint disease characterized by the degeneration of articular cartilage and the inflammation of synovium (synovitis) ${ }^{14)}$, and is a target disease of glucosamine ${ }^{4)-6}$.

Knee is one of the large joints in the body, and the ends of femur and tibia are coated with cartilage ${ }^{14)}$. Cartilage contains glycosaminoglycans and type II collagen, and acts like a shock absorber to spread the load more evenly across the joint. Moreover, a join capsule, called synovium, secretes synovial fluid containing hyaluronic acid, and synovial fluid helps the movement of the joint (Figure-5).

When a joint develops osteoarthritis, the cartilage gradually become damaged so the joint doesn't move as smoothly as it should. Under these pathological conditions, glycosaminoglycans and type II collagen in the cartilage are degraded ${ }^{14)}$. Moreover, the bone at the edge of the joint grows outwards, forming bony spurs called osteophytes, and the synovium makes extra fluid, although the content of hyaluronic acid is reduced. These pathological changes sometimes cause the pain, stiffness and swelling of the joint.

To evaluate the effect of glucosamine on osteoarthritis, we utilized a rat anterior cruciate ligament transection (ACLT) model as an osteoarthritis model ${ }^{15)}$. A knee joint is stabilized by collateral ligament, posterior cruciate and anterior cruciate ligaments. By ACLT, osteoarthritis is developed due to instability of the knee joint, accompanied with the degeneration of cartilage.

ACLT clearly induced the erosive change in the articular cartilage ${ }^{16)}$. Interestingly, glucosamine administration markedly suppressed the degenerative changes of the cartilage. Moreover, ACLT microscopically induced the surface depletion and reduced toluidine blue staining of proteoglycans in the cartilage. Importantly, glucosamine administration suppressed the surface depletion and proteoglycan degeneration in the cartilage.

Further, we evaluated the effect of glucosamine on the type II collagen-degradation and-synthesis markers ${ }^{17)}$. The level of CTX-II, a type II collagen- 
degradation marker, was elevated by ACLT. Importantly, glucosamine administration significantly suppressed the increase. Moreover, the level of CPII, a type II collagen-synthesis marker, was apparently increased by the administration of glucosamine to ACLT rats. These observations clearly indicate that glucosamine suppresses the type II collagen degradation but increases the type II collagen synthesis in ACLT model.

In addition, we evaluated the effect of glucosamine on the expression of matrix metalloprotease (MMP) -13, a collagen-degrading enzyme in the cartilage. The expression of MMP-13 was induced by ACLT, but was suppressed by glucosamine administration.

To further evaluate the chondroprotective action of glucosamine, human chondrocytes and synovial cells were incubated with glucosamine, and the production of glycosaminoglycans such as hyaluronic acid was determined ${ }^{18)}{ }^{19)}$. The results indicated that glucosamine increased the production of hyaluronic acid by chondrocytes and synovial cells.

Furthermore, the effect of glucosamine on the expression of hyaluronic acid-synthesizing enzymes (HAS) was evaluated by reverse transcriptase-polymerase chain reaction (RT-PCR). The results indicated that glucosamine increased the expression of HAS2 and HAS3 in chondrocytes, and HAS1 in synovial cells.

In recent years, sirtuins are hypothesized to play a key role in an organism's response to stresses (such as heat or starvation) and to be responsible for the lifespan-extending effects in yeast ${ }^{20)}$, nematodes $^{21)}$, insects ${ }^{22)}$ and mammalian cells ${ }^{23)}$.
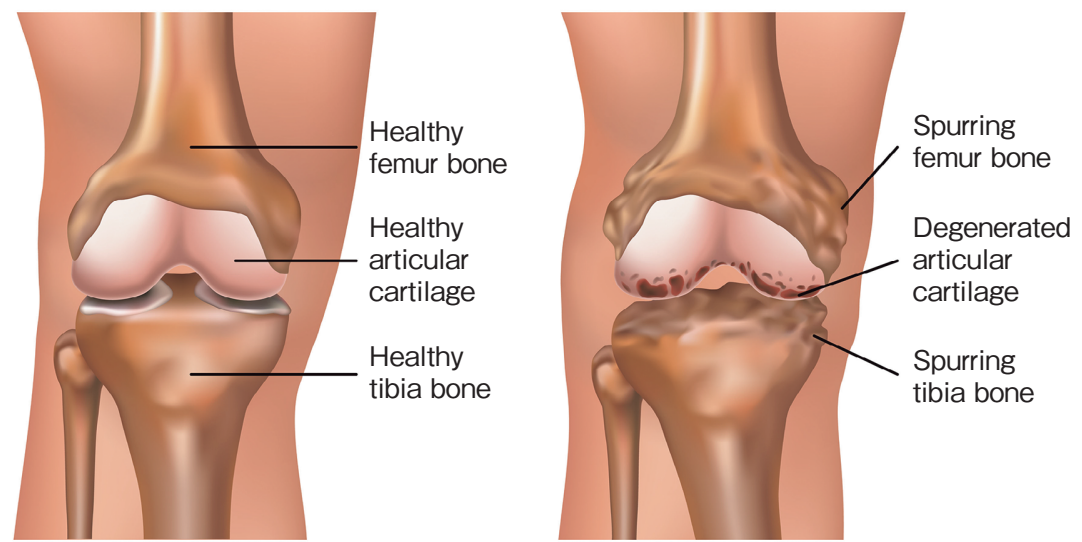

Interestingly, SIRT1, a member of sirtuin family, is suggested to act as a chondroprotective molecule in the cartilage. For example, SIRT1 protein is downregulated in chondrocytes from osteoarthritis patients, compared with normal individuals ${ }^{24)}$. Furthermore, heterozygous Sirt1 mice show the reduction of proteoglycans, type II collagen and aggrecan, and develop more severe osteoarthritis, compared with wild-type mice ${ }^{25}$. These findings strongly indicate that SIRT1 plays a crucial role in normal cartilage metabolism, and the reduction of SIRT1 leads to the development of osteoarthritis. Thus, we hypothesized that glucosamine may activate sirtuins thereby exhibiting a chondroprotective action.

To confirm this hypothesis, we incubated human chondrocytes with glucosamine, and the expression of sirtuin genes and proteins were analyzed by RT-PCR and western blotting, respectively ${ }^{26)}$. The results indicated that glucosamine apparently increased the expression of SIRT1 mRNA among sirtuin genes (SIRT1 7). Moreover, glucosamine significantly increased the protein level of SIRT1 in chondrocytes.

Figure- 6 shows the summary of the chondroprotective actions of glucosamine. First, the results with an ACLT model indicate that glucosamine exerts a chondroprotective action by inhibiting type II collagen degradation via the downregulation of MMP, and enhancing type II collagen synthesis in the articular cartilage.

Second, the in vitro study using chondrocytes and synovial cells indicate that glucosamine upregulates HAS and induces the production of hyaluronic acid,

Figure-5 Osteoarthritis of the knee (from http://www.dreamstime.com) 


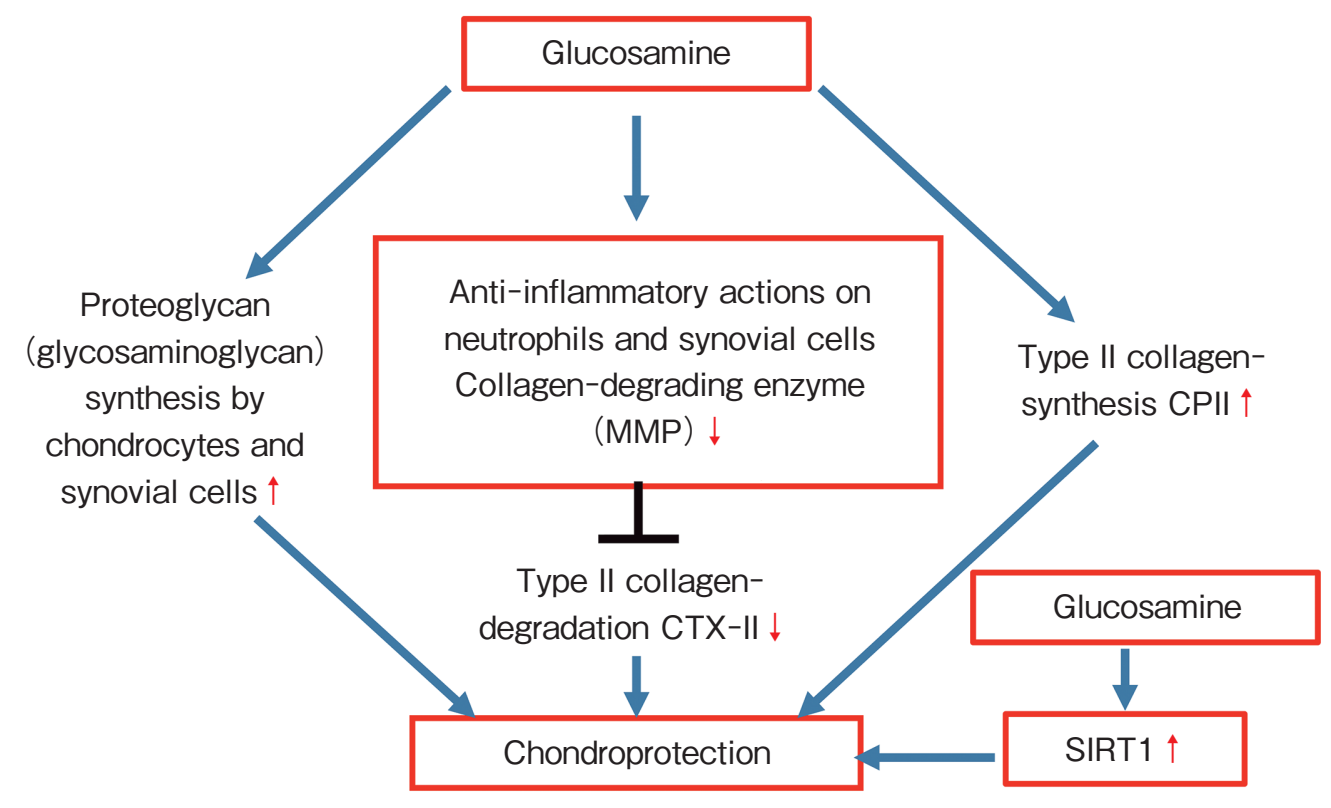

Figure-6 Schematic presentation of the chondroprotective actions of glucosamine

a major component of glycosaminoglycans, by chondrocytes and synovial cells.

Finally, glucosamine can upregulate the expression of SIRT1 in chondrocytes, thereby possibly exhibiting the chondroprotective action.

\section{Anti-inflammatory actions of glucosamine}

To investigate an anti-inflammatory action of glucosamine, we determined the effect of glucosamine on the activation of human synovial cells in vitro ${ }^{9)}$. Synovial cells were stimulated with IL- $1 \beta$ in the absence or presence of glucosamine, and IL- 8 (an inflammatory chemokine) was quantified by enzyme-linked immunoassay (ELISA). The results indicated that glucosamine suppressed the IL- $1 \beta^{-}$ induced production of IL-8 by synovial cells ${ }^{27}$. Similarly we revealed that glucosamine inhibits the THF- $\alpha$-induced activation of endothelial cells (the expression of an adhesion molecule ICAM-I, intercellular adhesion molecule-1 and a chemokine MCP-1, monocyte chemotactic protein-1) ${ }^{10}$.

Next, the anti-inflammatory mechanism of glucosamine was clarified. It is recognized that modification of cellular proteins with $O-\mathrm{N}$-acetylglucosamine $(O-G l c N A c)$ is involved in the modulation of cellular functions, such as signal transduction and transcription ${ }^{28)}$ (Figure-7). In this system, glucosamine is incorporated into the cells and converted to UDP-N-acetylglucosamine. Then, $\mathrm{N}$-acetylglucosamine (GlcNAc) is added to the serine or threonine residues of target proteins by $O$-GlcNAc transferase (OGT), and this reaction can be inhibited by alloxan, an $O-$ GlcNAc transferase inhibitor. Thus, the involvement of $O-G l c N A c$ modification in the action of glucosamine was investigated using alloxan ${ }^{27)}$. For this purpose, synovial cells were incubated with glucosamine in the absence or presence of alloxan, and $O^{-} \mathrm{GlcNAc}^{-}$ modified proteins were detected by western blotting with anti-O-GlcNAc antibody. The results indicated that glucosamine apparently increased the $O$-GlcNAc-modified proteins in synovial cells, and alloxan prevented the $O-$ GlcNAc modification. Furthermore, the effect of alloxan on IL-8 production was examined. Importantly, alloxan canceled the glucosamine-induced suppression of IL-8 production by synovial cells. Thus, the levels of $O$-GlcNAc modification were negatively correlated with the production of IL-8, when the cells were incubated with glucosamine in the absence or presence of alloxan.

Based on these findings, we are hypothesizing that glucosamine inhibits the synovial cell activation, such as IL-8 production via the $O-G l c N A c$ modification of target protein ( $\mathrm{s}$ ) (Figure-8). In contrast, alloxan prevents the $O$-GlcNAc modification, and restores the IL- 8 production. 


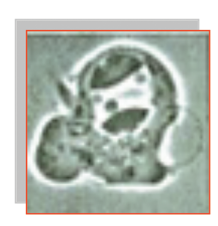

Signal transduction

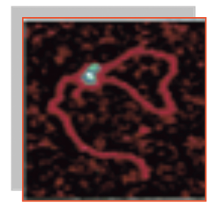

Transcription

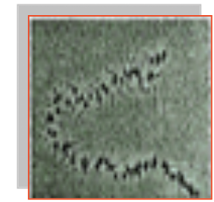

Translation

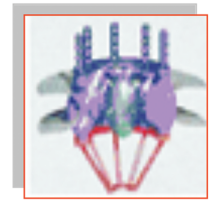

Transport

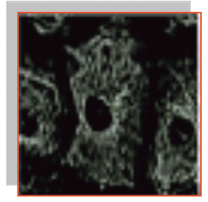

Cell shape

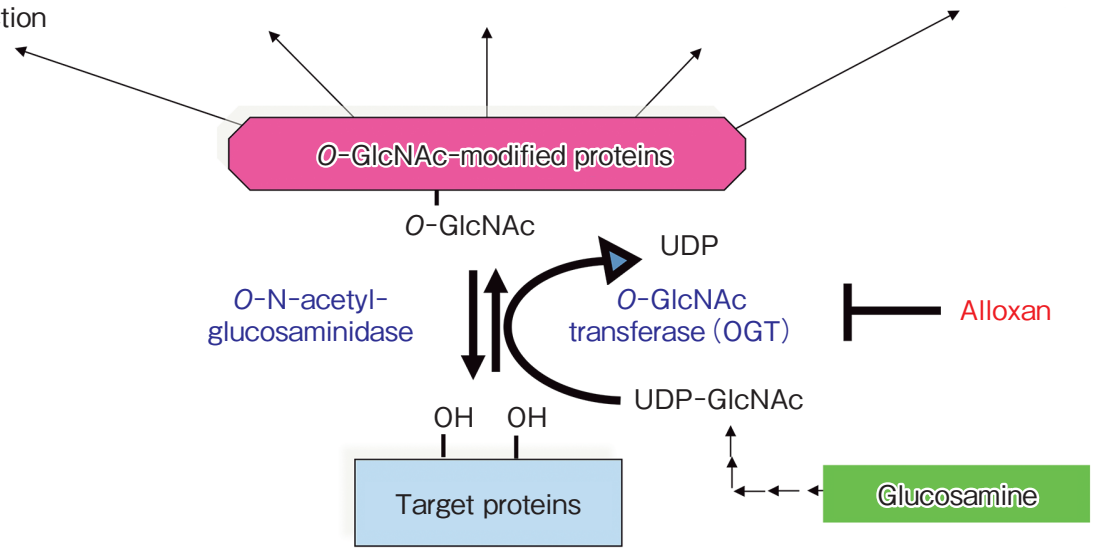

Figure-7 Roles of $O-\mathrm{N}$-acetylglucosamine $(O-\mathrm{GlcNAc})$-modification in cellular functions

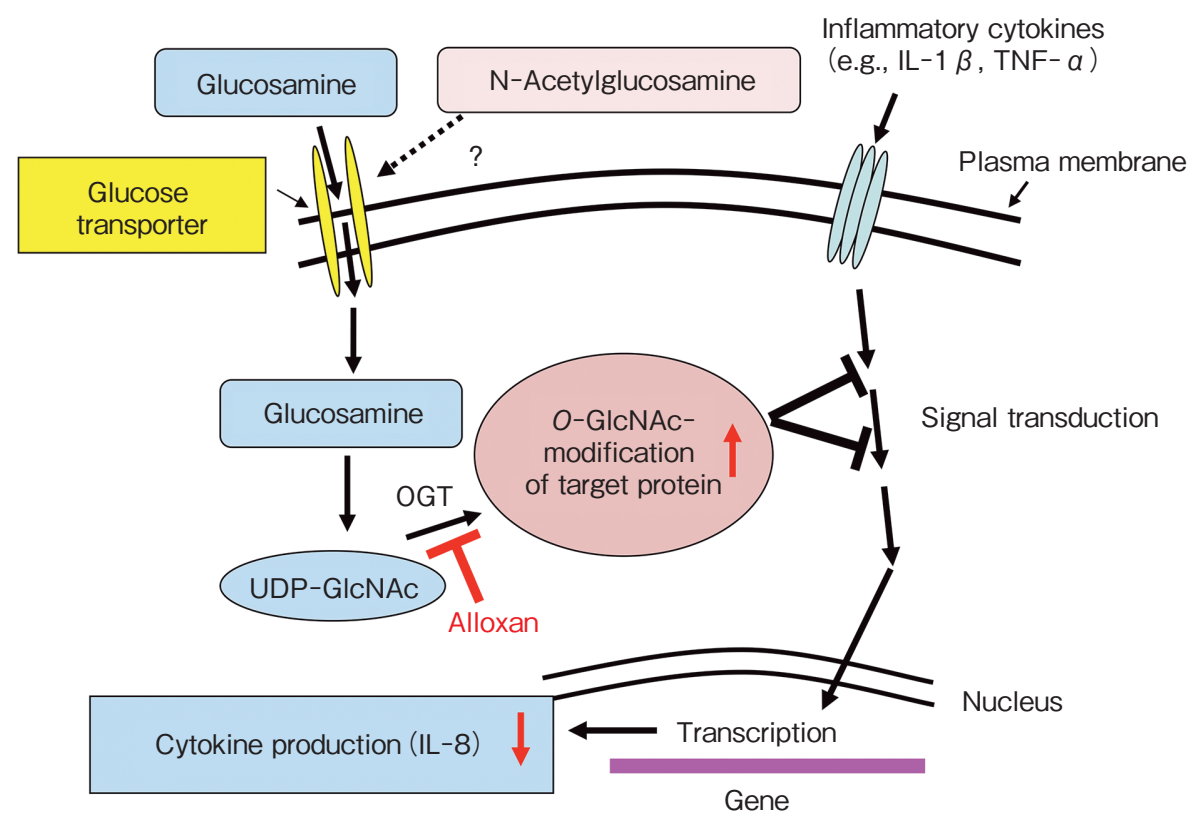

Figure-8 Possible mechanism for the $O$-GlcNAc modification-mediated suppression of cell activation by glucosamine

Furthermore, we tried to identify the $O-\mathrm{GlcNAc}^{-}$ modified target protein, which is involved in the anti-inflammatory action of glucosamine ${ }^{27)}$. For this purpose, synovial cells were incubated with glucosamine, and the cell lysates were subjected to immunoprecipitation with anti-O-GlcNAc antibody, and analyzed with sodium dodecyl sulfatepolyacrylamide gel electrophoresis (SDS-PAGE) analysis and mass spectrometry. The results indicated that $O_{-}^{-G l c N A c-m o d i f i e d ~ p r o t e i n s ~ i m m u-~}$ noprecipitated with anti-O-GlcNAc antibody were increased by glucosamine, and among a large number of proteins analyzed, a transcription factor Spl was identified as an $O$-GlcNAc-modified protein by mass spectrometry.

It has been already reported that $\mathrm{Sp} 1$ regulates the gene expression of cytokines such as IL-8 and $\mathrm{TNF}-\alpha^{29)}$, , and the transcriptional activity of Sp1 


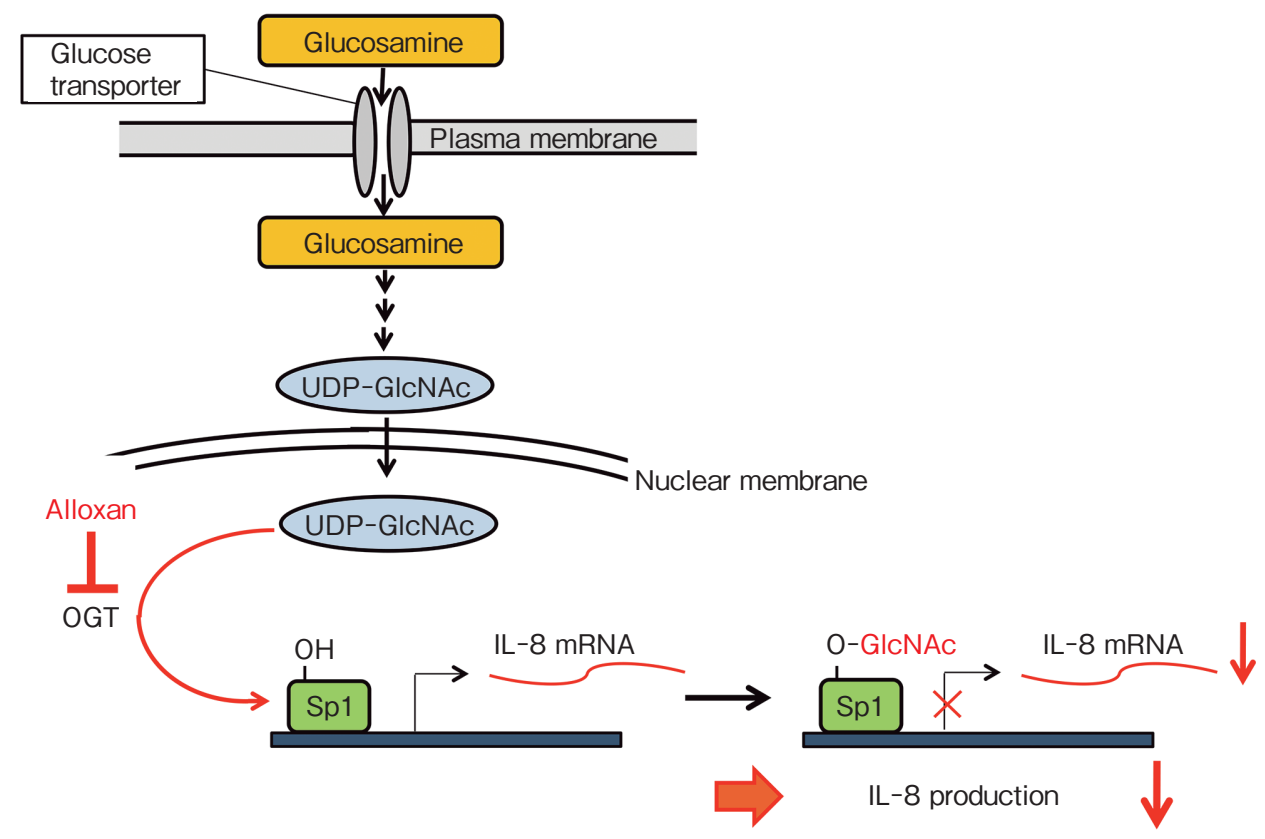

Figure-9 Mechanism for the suppression of IL-8 production by glucosamine-induced $O$-GlcNAc modification of Sp1

is reduced by $O-$ GlcNAc modification ${ }^{31}{ }^{32}$. So, we speculated that glucosamine downregulates the expression of cytokine genes via the $O-G l c N A c$ modification of Sp1. To confirm this possibility, $O-$ GlcNAc modification of SP1 was evaluated by western blotting using immunoprecipitated Sp1. The results indicated that glucosamine increased the $O$-GlcNAc modification of SP1, and alloxan effectively prevented this modification.

Based on these observations, we are hypothesizing that glucosamine is incorporated into the cells and induces the $O-\mathrm{GlcNAc}$ modification of Sp1 via the action of $O-G l c N A c$ transferase, and suppresses the transcription and production of IL-8 (Figure-9). In contrast, alloxan cancels the action of glucosamine by inhibiting $O-$ GlcNAc transferase, and restores the production of IL-8.

\section{Conclusions}

Our recent studies revealed the following observations.

1. Glucosamine normalizes the cartilage metabolism by inhibiting the degradation and enhancing the synthesis of type II collagen and glycosaminoglycans (such as hyaluronic acid). Moreover, glucosamine increases the expression of SIRT1 (a putative gene for the normal cartilage metabolism) in chondrocytes, thereby possibly exhibiting the chondroprotective action.

2. Glucosamine exerts the anti-inflammatory action via the $O-G l c N A c-$ modification of target proteins. A transcription factor $\mathrm{Sp} 1$ has been identified as an $O$-GlcNAc-modified protein, and the modification of SP1 is involved in the down-regulation of $\mathrm{IL}-8$ expression in synovial cells.

Thus, the present observations support the idea that glucosamine can be utilized therapeutically as a functional molecule with chondroprotective and anti-inflammatory actions in our body.

\section{Acknowledgments}

Drs. Akimasa Someya, Mamoru Igarashi and Jian Hua (Department of Host Defense and Biochemical Research, Juntendo University), Dr. Kiyohito Naito and Professor Kazuo Kaneko (Departments of Medicine for Motor Organs, Juntendo University), and Dr. Koji Sakamoto (Koyo Chemical Co.) are gratefully acknowledged for their discussions regarding the concepts of chondroprotective and anti-inflammatory actions of glucosamine.

\section{References}

1) Anderson JW, Nicolosi RJ, Borzelleca JF: Glucosamine 
effects in humans: a review of effects on glucose metabolism, side effects, safety considerations and efficacy. Food Chem Toxicol, 2005; 43: 187-201.

2) Esko JD, Kimata K, Lindahl U: Chapter 16. Proteoglycans and Sulfated Glycosaminoglycans. In: Varki A, Cummings RD, Esko JD, et al, eds. Essentials of Glycobiology, 2nd edition. New York: Cold Spring Harbor, 2009. http://www.ncbi.nlm.nih.gov/books/NBK1900

3) Glycosaminoglycans and Proteoglycans, The Medical Biochemistry Page, http://themedicalbiochemistrypage. org

4) Deal CL, Moskowitz RW: Nutraceuticals as therapeutic agents in osteoarthritis. The role of glucosamine, chondroitin sulfate, and collagen hydrolysate. Rheum Dis Clin North Am, 1999; 25: 379-395.

5) Reginster JY, Deroisy R, Rovati LC: Long-term effects of glucosamine sulphate on osteoarthritis progression: a randomised, placebo-controlled clinical trial. Lancet, 2001; 357: 251-256.

6) Pavelká K, Gatterová J, Olejarová M, et al: Glucosamine sulfate use and delay of progression of knee osteoarthritis: a 3-year, randomized, placebo-controlled, doubleblind study. Arch Intern Med, 2002; 162: 2113-2123.

7) Hua J, Sakamoto K, Nagaoka I: Inhibitory actions of glucosamine, a therapeutic agent for osteoarthritis, on the functions of neutrophils. J Leukoc Biol, 2002; 71: 632-640.

8) Hua J, Sakamoto K, Kikukawa T, Abe C, Kurosawa H, Nagaoka I: Evaluation of the suppressive actions of glucosamine on the interleukin- $1 \beta$-mediated activation of synoviocytes. Inflamm Res, 2007; 56: 432-438.

9) Yomogida S, Hua J, Sakamoto K, Nagaoka I: Glucosamine suppresses the interleukin- 8 production and ICAM- 1 expression by TNF- $\alpha$-stimulated human colonic epithelial cell line HT-29. Int J Mol Med, 2008; 22: 205-211.

10) Ju Y, Hua J, Sakamoto K, Ogawa H, Nagaoka I: Modulation of TNF- $\alpha$-induced endothelial cell activation by glucosamine, a naturally occurring amino monosaccharide. Int J Mol Med, 2008; 22: 809-815.

11) Hua J, Suguro S, Hirano S, Sakamoto K, Nagaoka I: Preventive actions of a high dose of glucosamine on adjuvant arthritis in rats. Inflamm Res, 2005; 54: 127132.

12) Yomogida S, Kojima Y, Tsutsumi-Ishii Y, Hua J, Sakamoto K, Nagaoka I: Glucosamine, a naturally occurring amino monosaccharide, suppresses dextran sulfate sodium-induced colitis in rats. Int J Mol Med, 2008; 22: 317-323.

13) Yomogida S, Kojima Y, Hua J, Ju Y, Sakamoto K, Nagaoka I: Evaluation of the effect of glucosamine on atherosclerosis using atherosclerotic $\mathrm{B} 6 \cdot \mathrm{KOR} \cdot \mathrm{Apoe}^{\mathrm{shl}}$ mice. Chitin Chitosan Res, 2008; 14: 55-61.

14) Osteoarthritis, Arthritis Research UK, http://www.ar thritisresearchuk.org

15) Hayami T, Pickarski M, Zhuo Y, Wesolowski GA, Rodan GA, Duong le T: Characterization of articular cartilage and subchondral bone changes in the rat anterior cruciate ligament transection and meniscectomized models of osteoarthritis. Bone, 2006; 38: 234-243.

16) Naito K, Watari T, Furuhata A, et al: Evaluation of the effect of glucosamine on an experimental rat osteoarthritis model. Life Sci, 2010; 86: 538-543.

17) Rousseau JC, Delmas PD: Biological markers in osteoarthritis. Nat Clin Pract Rheumatol, 2007; 3: 346-356.

18) Igarashi M, Kaga I, Takamori Y, Sakamoto K, Miyazawa K, Nagaoka I: Effects of glucosamine-derivatives and uronic acids on the production of glycosaminoglycans by human synovial cells and chondrocytes. Int J Mol Med, 2011; 27: 821-827.

19) Nagaoka I, Igarashi M, Sakamoto K: Biological activities of glucosamine and its related substances. Adv Food Nutr Res, 2012; 65: 337-352.

20) Howitz KT, Bitterman KJ, Cohen HY, et al: Small molecule activators of sirtuins extend Saccharomyces cerevisiae lifespan. Nature, 2003; 425: 191-196.

21) Tissenbaum HA, Guarente L: Increased dosage of a sir-2 gene extends lifespan in Caenorhabditis elegans. Nature, 2001; 410: 227-230.

22) Rogina B, Helfand SL: Sir2 mediates longevity in the fly through a pathway related to calorie restriction. Proc Natl Acad Sci USA, 2004; 101: 15998-16003.

23) Cohen HY, Miller C, Bitterman KJ, et al: Calorie restriction promotes mammalian cell survival by inducing the SIRT1 deacetylase. Science, 2004; 305: 390-392.

24) Dvir-Ginzberg M, Gagarina V, Lee EJ, Hall DJ: Regulation of cartilage-specific gene expression in human chondrocytes by SirT1 and nicotinamide phosphoribosyltransferase. J Biol Chem, 2008; 283: 36300-36310.

25) Gabay O, Oppenhiemer H, Meir H, Zaal K, Sanchez C, Dvir-Ginzberg M: Increased apoptotic chondrocytes in articular cartilage from adult heterozygous SirT1 mice. Ann Rheum Dis, 2012; 71: 613-616.

26) Igarashi M, Suzuki K, Sakamoto K, Nagaoka I: Sirtuin 1 is a target gene of glucosamine in chondrocytes. Glucosamine Res, 2013; 9: 21-24.

27) Someya A, Sakamoto K, Nagaoka I: Glucosamine induces the $\mathrm{O}-\mathrm{N}$-acetylglucosamine modification of transcription factor Sp1. Glucosamine Res, 2013; 9: 48-52.

28) Hanover JA: Glycan-dependent signaling: O-linked $\mathrm{N}$-acetylglucosamine. FASEB J, 2001; 15: 1865-1876.

29) Tsai EY, Falvo JV, Tsytsykova AV, et al: A lipopolysaccharide-specific enhancer complex involving Ets, Elk-1, Sp1, and CREB binding protein and p300 is recruited to the tumor necrosis factor alpha promoter in vivo. Mol Cell Biol, 2000; 20: 6084-6094.

30) Christofferson DE, Li Y, Hitomi J, et al: A novel role for RIP1 kinase in mediating TNF $\alpha$ production. Cell Death Dis, 2012; 3: e320.

31) Kudlow JE: Post-translational modification by O-GlcNAc: another way to change protein function. J Cell Biochem, 2006; 98: 1062-1075.

32) Lim K, Chang HI: O-GlcNAc inhibits interaction between Spl and Elf-1 transcription factors. Biochem Biophys Res Commun, 2009; 380: 569-574. 